\title{
NRAS NP_002515.1:p.G13V
}

National Cancer Institute

\section{Source}

National Cancer Institute. NRAS NP 002515.1:p.G13V. NCI Thesaurus. Code C155677.

A change in the amino acid residue at position 13 in the GT Pase NRas protein where glycine has been replaced by valine. 